\title{
EVOLUTION OF GALAXIES THROUGH THEIR INTERACTIONS
}

\author{
Y. FUNATO AND J. MAKINO \\ University of Tokyo, 3-8-1, Komaba, Meguro-ku, Tokyo, 153, \\ Japan
}

We investigated how the encounters between galaxies change their mass $M$ and velocity dispersion $\sigma$. We carried out a series of direct $N$-body simulation of encounters of two spherical galaxies. In Figure 1, the relative change of energy $\Delta E / E$ are plotted against that of mass $\Delta M / M$ for various initial conditions. The filled and open symbols correspond to the cases of Plummer model and relaxed Hernquist model, respectively. Here $\beta \equiv$ $(\Delta E / E) /(\Delta M / M)$.

We found a simple theory which explains the result. If the galaxies have halos with density $\rho \sim r^{-\alpha}$, the relation is expressed as $\Delta \sigma / \sigma=$ $0.25(\alpha-3) \Delta M / M$ independendly of the collisoin parameters.

For the real galaxies, $\alpha$ is believed to be 4 . Therefore the ratio $\beta$ is 0.25 . This implies that galaxies will assymptotically become to the state which is expressed as $\sigma \sim M^{0.25}$ through interactions. Assuming that $M / L$ is constant, this relation is equivalent to the Faber-Jackson relation $\sigma \sim L^{0.25}$.

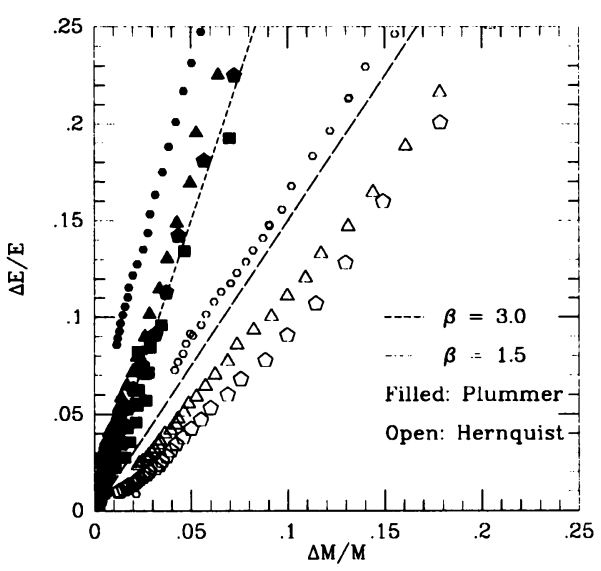

Figure 1. $\Delta E / E$ vs $\Delta M / M$ 